\title{
Decline in Child Vaccination Coverage During the COVID-19 Pandemic - Michigan Care Improvement Registry, May 2016-May 2020
}

\author{
Cristi A. Bramer, $\mathrm{MPH}^{1}$; Lynsey M. Kimmins, $\mathrm{MPH}^{1}$; Robert Swanson, $\mathrm{MPH}^{1}$; Jeremy Kuo, $\mathrm{MPH}^{1}$; Patricia Vranesich ${ }^{1,2}$; \\ Lisa A. Jacques-Carroll, MSW2; Angela K. Shen, ScD 2,3
}

\section{On May 18, 2020, this report was posted as an MMWR Early Release on the MMWR website (https://www.cdc.gov/mmwr).}

On March 13, 2020, the United States declared a national state of emergency to control the pandemic spread of SARS-CoV-2, the virus that causes coronavirus disease 2019 (COVID-19) (1). Public health response measures to mitigate the pandemic have centered on social distancing and quarantine policies, including shelter-in-place and stay-at-home orders. Michigan implemented a stay-at-home order on March 23, 2020, to facilitate social distancing (2). Such strategies might result in decreased accessibility to routine immunization services, leaving children at risk for vaccine-preventable diseases and their complications (3). To evaluate whether vaccination coverage has changed during the pandemic, data from the Michigan Care Improvement Registry (the state's immunization information system) (MCIR) were analyzed. Changes in vaccine doses administered to children and the effects of those changes on up-to-date status were examined for vaccinations recommended at milestone ages corresponding to the end of an Advisory Committee on Immunization Practices (ACIP) recommendation period for one or more vaccines (4).

The vaccination status of milestone age cohorts of children at ages 1, 3, 5, 7, 16, 19, and 24 months was assessed, with each cohort including an average sample size of 9,269 for the study period years 2016-2019, and 9,539 for 2020. Up-to-date status for individual vaccines and the recommended age-based vaccine series* were assessed at a point in time in May 2020 and compared with 1-month age cohort assessments for points in time in May 2016-May 2019. The number of noninfluenza vaccine doses administered and reported to MCIR for children aged $\leq 18$ years and aged $\leq 24$ months also were examined during January-April 2020, compared with averages for the same period in 2018 and 2019.

\footnotetext{
* Milestone age-based cohort assessments of recommended vaccine doses received were as follows: 1 month - 1st dose hepatitis B (HepB) within 3 days of life; 3 months - 2nd dose HepB, 1 rotavirus (Rota), 1 diphtheria, tetanus, and acellular pertussis (DTaP), 1 Haemophilus influenzae type b (Hib), 1 pneumococcal conjugate (PCV), 1 inactivated poliovirus (IPV); 5 months 2 HepB, 2 Rota, 2 DTaP, 2 Hib, 2 PCV, 2 IPV; 7 months - 2 HepB, up-to-date (UTD) Rota, 3 DTaP, UTD Hib, 3 PCV, 2 IPV; 16 months - 2 HepB, 3 DTaP, UTD Hib, 4 PCV, 2 IPV, 1 measles, mumps, rubella (MMR), 1 varicella (Var); 19 months -3 HepB, 4 DTaP, UTD Hib, 4 PCV, 3 IPV, 1 MMR, 1 Var; 24 months -3 HepB, 4 DTaP, UTD Hib, 4 PCV, 3 IPV, 1 MMR, 1 Var, 2 hepatitis A.
}

Vaccination coverage declined in all milestone age cohorts, except for birth-dose hepatitis B coverage, which is typically administered in the hospital setting (Figure). Among children aged 5 months, up-to-date status for all recommended vaccines declined from approximately two thirds of children during 2016-2019 (66.6\%, 67.4\%, 67.3\%, 67.9\%, respectively) to fewer than half (49.7\%) in May 2020. For the 16-month age cohort, coverage with all recommended vaccines declined, with measles-containing vaccination coverage decreasing from $76.1 \%$ in May 2019 to $70.9 \%$ in May 2020. In addition to a decline in up-to-date status in almost all age cohorts, the number of noninfluenza vaccine doses administered and reported for children aged $\leq 18$ years decreased $21.5 \%$, and the number of doses administered to children aged $\leq 24$ months decreased $15.5 \%$ during January-April 2020, compared with the same averaged periods in 2018 and 2019.

Up-to-date series coverage for each age cohort $(1,3,5,7$, 16, 19, and 24 months) assessed in May 2020 was lower for Medicaid-enrolled children than for those children not enrolled in Medicaid. The largest difference was in the age 7 months cohort assessed in May 2020; in that cohort, 34.6\% of Medicaidenrolled children were up-to-date for their recommended series, compared with 55.0\% of children not enrolled in Medicaid.

As the nation continues efforts to mitigate transmission of SARS-CoV-2, disruption of essential health services might occur, including in outpatient settings. Many provider offices have transitioned to telemedicine practices, where possible, to provide continuity of care in the medical home (5). Although some components of a well-child visit can be completed through telemedicine video conferences, immunization services require an in-person visit. Strategies to maintain immunization services include dedicating specific clinics, rooms, or buildings for sick visits and well visits; reducing the number of patients on-site at any one time; closing waiting rooms or registration areas, and having patients check in by phone and receive vaccinations from their vehicles in the parking lot $(\sigma)$. Providers can use their patients' electronic health records and immunization information systems to work with families to schedule in-person appointments, identify children who have missed recommended vaccinations, and assure parents that strict infection control practices are in place. The observed declines in vaccination coverage might leave young children and communities vulnerable to vaccine-preventable 
FIGURE. Percentage of Michigan infants and children vaccinated at milestone ages* — Michigan Care Improvement Registry, May 2016, 2017, 2018,2019 , and 2020

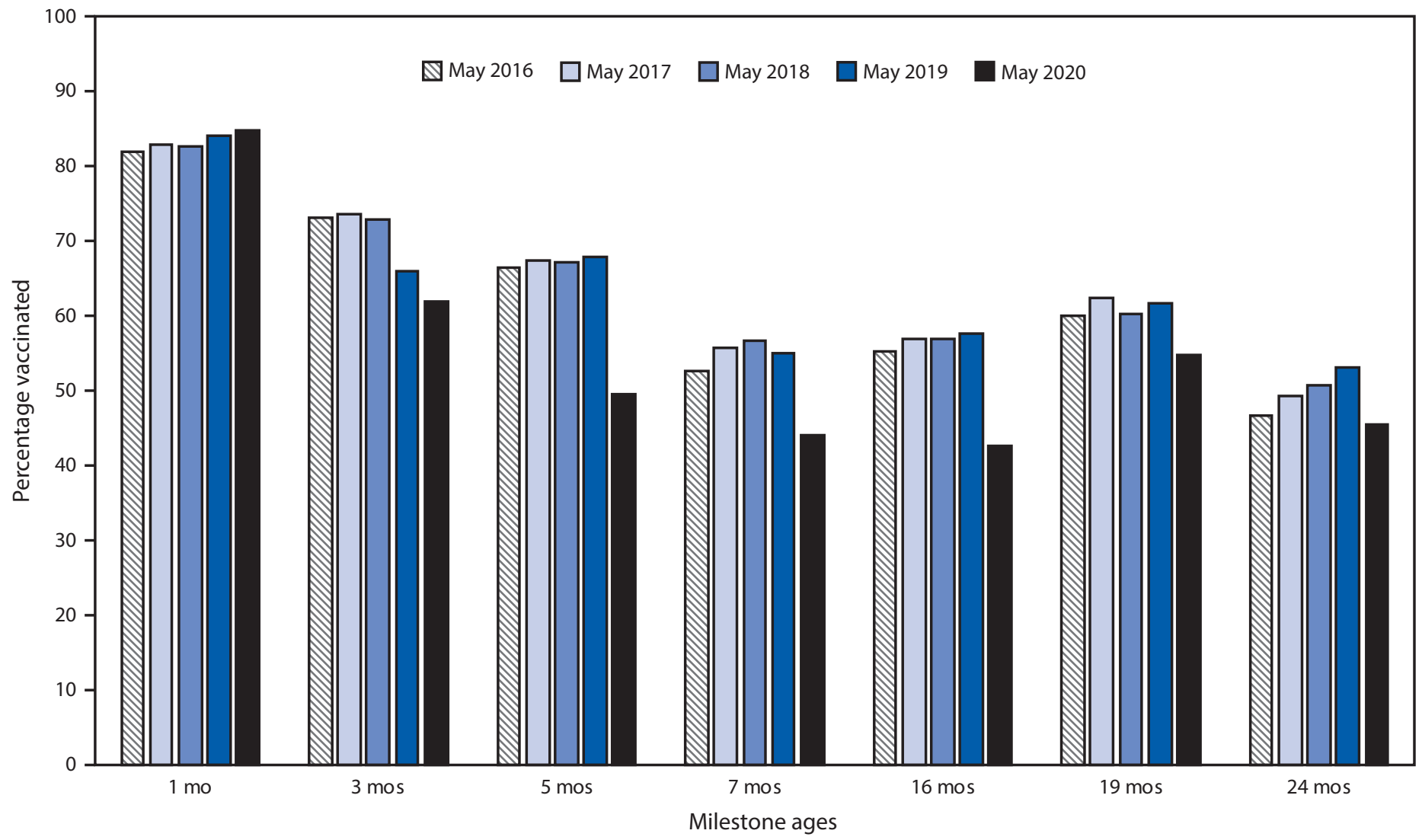

\begin{abstract}
* Milestone age cohorts (average sample size: 9,269 for 2016-2019, and 9,539 for 2020) were assessed at a point in time in May of each year. Milestone age-based cohort assessments of recommended vaccine doses received were as follows: 1 month - 1st dose hepatitis B (HepB) within 3 days of life; 3 months - 2nd dose HepB, 1 rotavirus (Rota), 1 diphtheria, tetanus, and acellular pertussis (DTaP), 1 Haemophilus influenzae type b (Hib), 1 pneumococcal conjugate (PCV), 1 inactivated poliovirus (IPV); 5 months - 2 HepB, 2 Rota, 2 DTaP, 2 Hib, 2 PCV, 2 IPV; 7 months - 2 HepB, up-to-date (UTD) Rota, 3 DTaP, UTD Hib, 3 PCV, 2 IPV; 16 months 2 HepB, 3 DTaP, UTD Hib, 4 PCV, 2 IPV, 1 measles, mumps, rubella (MMR), 1 varicella (Var); 19 months - 3 HepB, 4 DTaP, UTD Hib, 4 PCV, 3 IPV, 1 MMR, 1 Var; 24 months 3 HepB, 4 DTaP, UTD Hib, 4 PCV, 3 IPV, 1 MMR, 1 Var, 2 hepatitis A.
\end{abstract}

diseases such as measles. If measles vaccination coverage of $90 \%-95 \%$ (the level needed to establish herd immunity) is not achieved, measles outbreaks can occur. Concerted efforts are needed to ensure rapid catch-up for children who are not up-to-date with measles-containing vaccines as well as other ACIP-recommended vaccinations (4). Michigan continues to work with local health departments and vaccine providers to regularly assess patient populations for vaccination coverage, promote tools to conduct reminders and recalls, and develop provider and parent education regarding the continued need for vaccination during pandemics.

Corresponding author: Angela K. Shen, shenak@email.chop.edu, 301-467-7770.

${ }^{1}$ Division of Immunization, Michigan Department of Health and Human Services; ${ }^{2}$ Immunization Action Coalition, Saint Paul, Minnesota; ${ }^{3}$ Vaccine Education Center, Children's Hospital of Philadelphia, Philadelphia, Pennsylvania.

All authors have completed and submitted the International Committee of Medical Journal Editors form for disclosure of potential conflicts of interest. No potential conflicts of interest were disclosed.

\section{References}

1. CDC. Coronavirus disease 2019 (COVID-19). Cases in the U.S. Atlanta, GA: US Department of Health and Human Services, CDC; 2020. https:// www.cdc.gov/coronavirus/2019-ncov/cases-updates/cases-in-us.html

2. State of Michigan. Coronavirus. Stay home. Stay safe. Save lives. Lansing, MI: State of Michigan; 2020. https://www.michigan.gov/ coronavirus/0,9753,7-406-98159-52264--,00.html

3. Santoli JM, Lindley MC, DeSilva MB, et al. Effects of the COVID-19 pandemic on routine pediatric vaccine ordering and administrationUnited States, 2020. MMWR Morb Mortal Wkly Rep 2020;69:591-3. https://doi.org/10.15585/mmwr.mm6919e2

4. CDC. Recommended child and adolescent immunization schedule for ages 18 years or younger, United States, 2020. Atlanta, GA: US Department of Health and Human Services, CDC; 2020. https://www. cdc.gov/vaccines/schedules/hcp/imz/child-adolescent.html

5. American Academy of Family Physicians. Inside look at using telemedicine during COVID-19 pandemic, 2020. Leawood, KS: American Academy of Family Physicians; 2020. https://www.aafp.org/news/health-of-thepublic/20200323covidtelehealth.html

6. Washington State Department of Health. Please continue vaccinating patients during COVID-19. Seattle, WA: Washington State Department of Health; 2020. https://files.constantcontact.com/9817310a001/66cddbb8c4f9-44b4-a762-20e04beaaed3.pdf 